\title{
Unexpected behaviour of cross sections of high energy protons
}

\author{
I.M. Dremin ${ }^{1,2, a}$ \\ 1 Lebedev Physics Institute, Moscow 119991, Russia \\ 2 National Research Nuclear University "MEPhI", Moscow 115409, Russia
}

\begin{abstract}
Nobody expected that protons will try to keep their entity in their collisions at higher and higher energies. However, the comparison of experimental results on proton-proton interactions from ISR to LHC energies clearly shows this tendency with a definitely increasing share of the elasic scattering cross section. This unexpected behaviour immediately leads to the unexpected corollary about the shape of the spatial interaction region of the two protons. The unquestionable principle of unitarity combined with available experimental data on elastic scattering is used to get new conclusions about this shape. Its evolution at present energies from the ISR to the LHC and predictions about its behaviour at ever higher energies are considered. The shape can transform rather drastically if the share of elastic processes keeps rising. The possible origin of the effect and its interrelation to the strong interaction dynamics are speculated. Cosmic ray studies can help in clarifying this behaviour.
\end{abstract}

Experimental data on proton-proton interactions in high energy collisions show quite a special and unexpected behaviour of the share of elastic scattering compared to inelastic processes with increasing energy. It decreases at the beginning (at comparatively low energies) but then starts to increase. From Intersecting Storage Rings (ISR) energies of $20-60 \mathrm{GeV}$ up to higher energies $7-13 \mathrm{TeV}$ at the Large Hadron Collider (LHC) it increases by more than a factor 1.5! According to intuitive classical ideas we would expect a stable tendency with increasing share of the break-down of protons compared to their survival probability.

If a cup falls to the floor, it breaks into pieces but sometimes stays intact. The harder it hits the floor, the less chance to be unbroken.

If two high energy protons collide, many new particles (mostly pions) are produced, but sometimes they scatter elastically and retain their entity. It is surprising enough that at very high collision energies the proportion of elastic processes increases with increasing energy from the ISR to the LHC.

One can assume that either the asymptotic freedom or the extremely short time of flight of high energy protons through each other are in charge of such a surprising effect. The unquestionable principle of unitarity combined with the available experimental data on elastic scattering is used to get new conclusions about the shape of the interaction region of colliding protons. Its evolution at present energies is considered. Some predictions about its behaviour at even higher energies are described with different assumptions on the relative roles of elastic scattering and inelastic processes. The shape can transform rather drastically if the proportion of elastic processes keeps rising. This unexpected property leads to an unexpected corollary. The possible origin of the effect

a e-mail: dremin@td.lpi.ru and its interrelation to the strong interaction dynamics are speculated.

Our main goal here is to get some knowledge about the spatial region of interactions of high energy protons at current energies, to draw a pictorial view of its evolution with increasing energy and to discuss possible theoretical and experimental implications of these findings.

The most stringent and reliable information (albeit rather limited!) about the interrelation of elastic and inelastic processes comes from the unitarity of the $S$ matrix

$$
S S^{+}=1
$$

or for the scattering matrix $T(S=1+i T)$

$$
2 \operatorname{Im} T_{a b}=\Sigma_{n} \int T_{a n} T_{n b}^{*} d \Phi_{n},
$$

where $a, b, n$ denote the number of particles. The whole $n$-particle phase space $\Phi_{n}$ is integrated over. For the elastic scattering amplitude $a=b=2$, the unitarity condition relates the amplitude of elastic scattering $f \propto T_{22}$ to the amplitudes of $n$-particle inelastic processes $T_{2 n}$ declaring that the total probability of all outcomes of the interaction (elastic and inelastic) must be equal to $1^{1}$. Information about the elastic scattering of protons comes from the measurement of the differential cross section $d \sigma / d t$ at some energy, $s$, as a function of the transferred momentum, $t$, at its experimentally accessible values. It is related to the scattering amplitude, $f(s, t)$, in the following way

$$
\frac{d \sigma}{d t}=|f(s, t)|^{2}
$$

The variables $s$ and $-t$ are respectively the squared total energy, $2 E$, and squared transferred momentum of

\footnotetext{
${ }^{1}$ The non-linear contribution from the elastic amplitude appears in the right-hand side for $n=2$.
} 
Table 1. The energy behaviour of $\zeta, G(s, 0)$ and $\sigma_{\text {inel }} / \sigma_{e l}$.

\begin{tabular}{|l|l|l|l|l|l|l|l|l|}
\hline$\sqrt{s}, \mathrm{GeV}$ & 4.11 & 4.74 & 7.62 & 13.8 & 62.5 & 546 & 1800 & 7000 \\
\hline$\zeta$ & 0.98 & 0.92 & 0.75 & 0.69 & 0.67 & 0.83 & 0.93 & $1.00-1.04$ \\
$G(s, 0)$ & 1.00 & 0.993 & 0.94 & 0.904 & 0.89 & 0.97 & 0.995 & 1.00 \\
\hline & & & & & ISR & $\mathrm{S} p \bar{p} \mathrm{~S}$ & FNAL & LHC \\
\hline$\sigma_{\text {inel }} / \sigma_{e l}$ & & & & & $\mathbf{5}$ & & & $\mathbf{3}$ \\
\hline
\end{tabular}

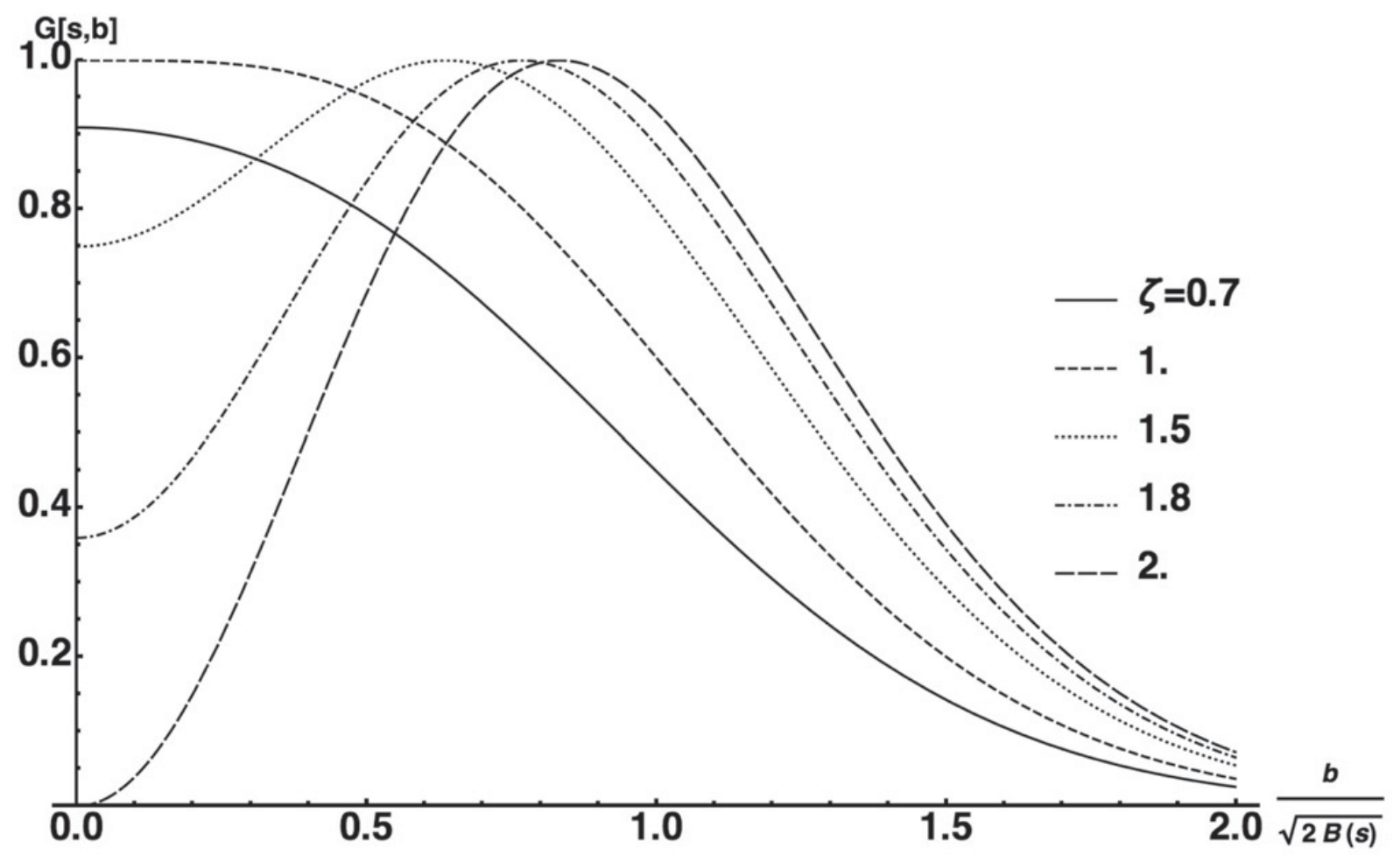

Figure 1. The energy evolution of the shape of the inelastic interaction region for different values of the survival probability $\zeta / 4$. The values $\zeta=0.7$ and 1.0 correspond to ISR and LHC energies and agree well with the result of detailed fitting to the elastic scattering data [4-6]. A further increase of $\zeta$ leads to the change of the BEL regime (Blacker, Edgier, Larger - [7]) by the toroid-like shape with a dip at $b=0$ (TEH regime-Toroidal Elastic Hollow- [8-11]) The values $\zeta=1.5$ are proposed in $[12,13]$ and $\zeta=1.8$ in [14] as describing the asymptotical regimes. The value $\zeta=2$ corresponds to the so-called "black disk" regime with equal elastic and inelastic cross sections $\left(\sigma_{e l}=\sigma_{\text {inel }}=0.5 \sigma_{\text {tot }}\right)$.

the two colliding protons in the center-of-mass system $s=$ $4 E^{2}=4\left(p^{2}+m^{2}\right)(p$ is the proton momentum $)$ and $-t=$ $2 p^{2}(1-\cos \theta)$ at the scattering angle, $\theta$. In the $s$ channel this unquestionable condition is usually expressed in the form of the well known integral relation (for more details see [1,2]). This relation is quite complicated for arbitrary values of the transferred momentum, $t$. However, for forward scattering at $t=0$ it leads to the widely used optical theorem showing the normalization of the imaginary part of the amplitude $\operatorname{Im} f(s)$ by its direct connection with the total cross section $\sigma_{\text {tot }}$ :

$$
\operatorname{Im} f(s, 0)=\sigma_{\text {tot }}(s) / 4 \sqrt{\pi}
$$

and to the general statement that the total cross section is the sum of cross sections of elastic and inelastic processes

$$
\sigma_{\text {tot }}=\sigma_{e l}+\sigma_{\text {inel }},
$$

i.e., that the total probability of all processes equals 1 .

One can use the Fourier - Bessel transform of the amplitude, $f$, to reduce the integral relation to the more simple algebraic one. This transformation retranslates the momentum data to the shortest transverse distance between the trajectories of the centers of colliding protons called the impact parameter, $b$, and is written as

$$
i \Gamma(s, b)=\frac{1}{2 \sqrt{\pi}} \int_{0}^{\infty} d|t| f(s, t) J_{0}(b \sqrt{|t|}) .
$$

Then the unitarity condition in the $b$-representation reads

$$
G(s, b)=2 \operatorname{Re} \Gamma(s, b)-|\Gamma(s, b)|^{2}
$$

(for reviews see, e.g., Refs. [2,3]). This relation establishes the connection between the distributions of the intensity of all processes in the transverse configuration space:

$$
\frac{d^{2} \sigma_{\text {inel }}}{d b^{2}}=\frac{d^{2} \sigma_{t o t}}{d b^{2}}-\frac{d^{2} \sigma_{e l}}{d b^{2}}
$$

The left-hand side in Eqs. (7), (8) describes the transverse impact-parameter profile of inelastic collisions of protons. It satisfies the inequalities $0 \leq G(s, b) \leq 1$ and determines how absorptive the interaction region is at the given impact parameter (with $G=1$ for the full absorption and $G=0$ for the complete dominance of elastic scattering). The profile of elastic processes is 
determined by the subtrahend in Eqs. (7), (8). Thus we get a spatial view of the whole process if the elastic scattering amplitude $f$ is integrated in Eq. (6).

In the following, we use the exponential parametrization of the imaginary part of the amplitude, $f$, to proceed with analytical calculations and argue that it is very precise:

$$
\operatorname{Im} f(s, t)=\frac{\sigma_{t o t}(s)}{4 \sqrt{\pi}} \exp [B(s) t / 2] .
$$

Formally, this approximation is not valid for differential cross sections at large transferred momenta. However, for our purposes only the integral contribution of $f$ at large $|t|$ to Eq. (6) is important. It is negligibly small there compared to the peak of the diffraction cone.

For central collisions $b=0$ the unitarity condition (7) is written as

$$
G(s, b=0)=\zeta(2-\zeta)
$$

where

$$
\zeta(s)=\frac{4 \sigma_{e l}(s)}{\sigma_{t o t}(s)} .
$$

Thus, according to the unitarity condition (Eq. 10) the darkness of the inelastic interaction region for central collisions (absorption) is defined by the only experimentally measured parameter $\zeta(s)$ depending on energy. It has the maximum $G(s, 0)=1$ for $\zeta=1$ (see Table 1 for the LHC and Figure 1). Any decline of $\zeta$ from $1(\zeta=1 \pm \epsilon)$ results in the parabolic decrease of the absorption $\left(G(s, 0)=1-\epsilon^{2}\right)$, i.e., to an even much smaller decline from 1 for small $\epsilon$. The elastic profile, equal to $\zeta^{2}$ in central collisions, also reaches the value 1 for $\zeta=1$.

Let us rewrite Eq. (10) as

$$
\zeta(s)=1 \pm \sqrt{1-G(s, 0)} .
$$

The critical value $\zeta=1$ reveals itself in the usage of different signs in front of the square root term (different branches of the unitarity condition) for $\zeta<1$ and $\zeta>1$.

The shape of the inelastic profile at any $b$ is given by

$$
G(s, b)=\zeta \exp \left(-\frac{b^{2}}{2 B}\right)\left[2-\zeta \exp \left(-\frac{b^{2}}{2 B}\right)\right]
$$

It is shown in Fig. 1 for different $\zeta$. The drastic change of the shape of the inelastic interaction region is seen. It becomes completely dark for central collisions up to LHC energies and can develop a hollow in there at higher energies. The elastic scattering of protons can become prevailing in the central region. It can have some implications for extensive air showers at energies above $10^{15} \mathrm{eV}$ in the laboratory system that correspond to ISR energies where the effect of the increasing share of elastic scattering becomes observable. The detailed analyses of such extensive air showers can, probably, say something about "escaping" high energy protons.

To conclude, it has been shown that an unexpected property leads to an unexpected corollary. This is the uncommon shape of the interaction region and the transition to another branch of the unitarity condition.

No reliable explanation of these findings exists yet.

\section{References}

[1] K.A. Olive (PDG Collaboration), China Phys. C 38, 090513 (2014)

[2] I.M. Dremin, Physics-Uspekhi 56, 3 (2013)

[3] I.M.Dremin, Physics-Uspekhi 58, 61 (2015)

[4] U. Amaldi et al., Phys. Lett. B 44, 112 (1973)

[5] I.M. Dremin, V.A. Nechitailo, Nucl. Phys. A 916, 241 (2013)

[6] A. Alkin, E. Martynov, O. Kovalenko, S.M. Troshin, Phys. Rev. D 89, 091501 (2014) (R)

[7] R.Henzi, P. Valin, Phys. Lett B 132, 443 (1983)

[8] I.M. Dremin, JETP Lett. 99, 243 (2014)

[9] I.M. Dremin, S.N. White, The interaction region of high energy protons; arXiv 1604.03469

[10] S.N. White, Talk at the conference QCD at Cosmic Energies, Chalkida, Greece, May 2016; http://www.lpthe.jussieu.fr/cosmic2016/TALKS/ White.pdf

[11] I.M. Dremin, Int. J. Mod. Phys. A 31, 1650107 (2016)

[12] A.K. Kohara, E. Ferreira, T. Kodama, Eur. Phys. J. C 74, 3175 (2014)

[13] D.A. Fagundes, M.J. Menon, P.V.R.G. Silva, Nucl. Phys. A 946, 194 (2016)

[14] S.M. Roy, A two component picture for high energy scattering: unitarity, analitycity and LHC data; arXiv:hep-ph, 1602.03627 